\title{
Computational pair density functional theory: A proposal for the kinetic energy functional
}

\author{
Katsuhiko Higuchi \\ Graduate School of Advanced Sciences of Matter, Hiroshima University, Higashi-Hiroshima 739-8527, Japan \\ Masahiko Higuchi \\ Department of Physics, Faculty of Science, Shinshu University, Matsumoto 390-8621, Japan \\ (Received 27 March 2010; revised manuscript received 27 September 2010; published 22 October 2010)
}

\begin{abstract}
We present a computational pair density (PD) functional theory using multiple Slater determinants in constructing the PD. Compared to the effective initial scheme [M. Higuchi and K. Higuchi, Phys. Rev. B 78, 125101 (2008)], the search region for the ground-state PD is substantially extended within the set of $N$-representable PDs. The merit of the present scheme is to guarantee the resultant PD to be $N$-representable and to describe correlation effects beyond the effective initial scheme, which enables us to tackle with the development of the approximate form of the kinetic energy functional. We derive two restrictive conditions on the kinetic energy functional. With the aid of these restrictive conditions, we also propose several types of approximate functionals of the kinetic energy. To check the abilities and limitations of the present scheme including such approximate functionals, actual calculations are performed for the neutral neon atom. The results show that the present scheme provides the $N$-representable and correlated PDs, though it would have some limitations. What we need to do first for improvement of the computational PD functional theory is that the development of the approximate functional of the kinetic energy is further advanced along the present scheme.
\end{abstract}

DOI: 10.1103/PhysRevB.82.155135

PACS number(s): 71.15.Mb

\section{INTRODUCTION}

The diagonal element of the second-order reduced density matrix, which is called the pair density (PD), has more information than the electron density. Not only the electron density but also expectation values of arbitrary two-particle operators can be obtained from the PD. ${ }^{1-3}$ Therefore, the density functional scheme, where the PD is chosen as a basic variable, has recently attracted much attentions as a potential scheme beyond the conventional density functional theory (DFT). ${ }^{4,5}$ This theory has been called PD functional theory.

The PD functional theory was first proposed by Ziesche. ${ }^{6,7}$ After his proposal, many workers have studied it extensively, ${ }^{8-41}$ so that we have comprehended that there exist two kinds of problems for the development of the PD functional theory.

First one is so-called $N$-representability problem of the PD. We have to carry out the variational principle with respect to the PD with varying it within the set of the $N$-representable PDs. 6,7,12,13,20 However, the necessary and sufficient conditions on the $N$-representability of the PD have not been obtained in a practical form. ${ }^{1-3,24-41}$ Therefore, it is difficult to search the ground-state PD within the correct set of $N$-representable PDs. Although several ways to avoid the first problem have been proposed, ${ }^{12,13,17,19,29}$ unfortunately actual calculations have not yet been performed. Whereas, we have previously presented the computational PD functional theory, in which the PD is constructed from the single Slater determinant (SD). ${ }^{20,22,23}$ This scheme is, of course, free from the $N$-representability problem. Furthermore, it has been formally shown that the correlation effects are covered more or less but definitely. Actually test calculations for the neutral neon atom show that the correlation energy is reproduced by about $20 \% .^{22,23}$ Thus, since it obviously provides the $N$-representable and correlated PD, this scheme can be positioned as an effective initial theory in the field of the PD functional theory, just like the Hartree-Fock approximation in the field of the wave-function theory. ${ }^{22}$ It is expected that the effective initial scheme can be a good starting point toward developing more sophisticated theory. In order to go beyond the effective initial scheme, we have to extend the search region over the set of the PDs constructed from the single SDs. ${ }^{42}$

The second problem for the development of the PD functional theory is related to the approximate form of the kinetic energy functional. As is well known, although the electronelectron interaction energy can be written in terms of the PD, the kinetic energy cannot exactly be written by using the PD alone. Some approximation is needed for the kinetic energy functional of the PD functional theory. Several workers have developed approximate forms so far. ${ }^{16,30,41,43}$ For instance, the kinetic energy functional of the PD functional theory is approximated by the sum of the kinetic energy that is calculated by the Kohn-Sham SD of the original DFT and the correction term. ${ }^{30}$ In the effective initial scheme, ${ }^{20,22,23}$ the kinetic energy of the noninteracting reference system has been utilized, and the correlating kinetic energy, the remaining term of the kinetic energy after deduction of the kinetic energy of the noninteracting reference system, has been approximated with the aid of the scaling property of the energy functional. ${ }^{20,22,23}$ Since the accuracy of the ground-state PD is directly dependent on the approximations of the kinetic energy, we have to devote additional efforts to the development of these ones.

In this paper, we attempt to tackle the above problems. Concerning the first problem, as mentioned above, we need to extend the search region of the PD with preserving the $N$-representability of the PD. We shall extend the search region by using multiple SDs in constructing PDs. The search region of the $\mathrm{PD}$ is absolutely extended compared to the 
effective initial scheme. Since the $N$-representability of the $\mathrm{PD}$ is guaranteed so far as the PD is constructed from the antisymmetric wave function, the $N$-representability of the present scheme is kept independently of the number of the SDs. This is a strong merit of the present scheme, which enables us to tackle with the second problem.

The strategy to the second problem is to utilize the exact relations and bounds of the kinetic energy functional as restrictive conditions on its approximate forms. This strategy is similar to that of the development of new energy functionals of the generalized gradient approximation, ${ }^{44-47}$ vorticity expansion approximation, ${ }^{48-51}$ and etc, which has been proved sound in these cases. In this paper, we derive two restrictive conditions on the kinetic energy functional by using the Hohenberg-Kohn theorems of the PD and the scaling property of the kinetic energy functional. Using these conditions, we propose several types of approximate forms of the kinetic energy functional. We also perform test calculations for checking the abilities and limitations of the present scheme including these approximate functionals. Based on the results of test calculations, we discuss the direction of advancing the computational PD functional theory.

Organization of this paper is as follows. In Sec. II, the effective equation for the ground-state PD that is constructed from the multiple SDs is derived with the use of the Hohenberg-Kohn theorems of the PD. In Sec. III, we derive restrictive conditions on the kinetic energy functional of the PD functional theory so as to develop its approximate forms. In Sec. IV, several types of approximate forms are presented. In order to evaluate the abilities and limitations of the present scheme including such approximate functionals, test calculations are performed for the neutral neon atom. The evaluation method is explained in Sec. V A. The details of numerical calculations are mentioned in Sec. V B. Although it provides the $N$-representable and correlated PDs, the present scheme has room for improvement. The relevant discussions are given in Sec. V C. In order to stress the position of the present scheme in the field of the PD functional theory, we mention the features and future works in Sec. V D. Finally, some concluding remarks are given in Sec. VI.

\section{PD FUNCTIONAL THEORY BY MEANS OF THE MULTIPLE SLATER DETERMINANTS}

In the present scheme, the PD is constructed from the multiple SDs so as to extend the search region more largely than the effective initial scheme. ${ }^{20,22,23}$ Using the variational principle with respect to the $\mathrm{PD}$, we derive the effective equation that gives an $N$-representable and correlated PD of the ground state.

\section{A. Expression for the PD}

The antisymmetric wave function is written as the linear combination of the SDs

$$
\Psi\left(\mathbf{r}_{1} \eta_{1}, \mathbf{r}_{2} \eta_{2}, \ldots, \mathbf{r}_{N} \eta_{N}\right)=\sum_{i} C_{i} \Phi_{i}\left(\mathbf{r}_{1} \eta_{1}, \mathbf{r}_{2} \eta_{2}, \ldots, \mathbf{r}_{N} \eta_{N}\right),
$$

where $\mathbf{r}_{j}$ and $\eta_{j}$ are spatial and spin coordinates of the $j$ th electron, respectively, and where $\Phi_{i}\left(\mathbf{r}_{1} \eta_{1}, \mathbf{r}_{2} \eta_{2}, \ldots, \mathbf{r}_{N} \eta_{N}\right)$ is the SD which is given by

$$
\begin{aligned}
& \Phi_{i}\left(\mathbf{r}_{1} \eta_{1}, \mathbf{r}_{2} \eta_{2}, \ldots, \mathbf{r}_{N} \eta_{N}\right) \\
& \quad=\frac{1}{\sqrt{N !}} \sum_{P}(-1)^{p} P \phi_{\alpha}^{i}\left(\mathbf{r}_{1} \eta_{1}\right) \phi_{\beta}^{i}\left(\mathbf{r}_{2} \eta_{2}\right) \cdots \phi_{\lambda}^{i}\left(\mathbf{r}_{N} \eta_{N}\right),
\end{aligned}
$$

where $P$ is a permutation operator upon the electronic coordinates, $p$ is the number of interchanges in $P$, and the constituent single-particle wave functions of the SD, i.e., $\phi_{\alpha}^{i}\left(\mathbf{r}_{1} \eta_{1}\right), \phi_{\beta}^{i}\left(\mathbf{r}_{2} \eta_{2}\right), \ldots$, are normalized and orthogonal to each other. The PD is defined as

$$
\begin{aligned}
\gamma^{(2)}\left(\mathbf{r} \mathbf{r}^{\prime} ; \mathbf{r r}^{\prime}\right)= & \frac{N(N-1)}{2} \int\left|\Psi\left(\mathbf{r} \eta, \mathbf{r}^{\prime} \eta^{\prime}, \mathbf{r}_{3} \eta_{3}, \ldots, \mathbf{r}_{N} \eta_{N}\right)\right|^{2} \\
& \times d \eta d \eta^{\prime} d^{3} r_{3} d \eta_{3} \cdots d^{3} r_{N} d \eta_{N} .
\end{aligned}
$$

Substituting Eq. (1) into Eq. (3), and using the cofactor expansion of the SD, the PD is written as

$$
\begin{aligned}
\gamma^{(2)}\left(\mathbf{r r}^{\prime} ; \mathbf{r r}^{\prime}\right)= & \sum_{i}\left|C_{i}\right|^{2} \gamma_{\mathrm{SSD}, i}^{(2)}\left(\mathbf{r r}^{\prime} ; \mathbf{r} \mathbf{r}^{\prime}\right) \\
& +\frac{1}{2} \sum_{i} \sum_{j}^{\prime} C_{i}^{*} C_{j} \Gamma_{i_{1} j_{1}}^{i j}\left(\mathbf{r r}^{\prime}\right) \\
& +\frac{1}{2} \sum_{i} \sum_{j}^{\prime \prime} C_{i}^{*} C_{j} \Gamma_{i_{1} i_{2} j_{1} j_{2}}^{i j}\left(\mathbf{r r}^{\prime}\right)
\end{aligned}
$$

with

$$
\begin{aligned}
& \gamma_{\mathrm{SSD}, i}^{(2)}\left(\mathbf{r} \mathbf{r}^{\prime} ; \mathbf{r r}^{\prime}\right)=\iint d \eta d \eta^{\prime} \frac{1}{2} \sum_{\alpha}^{N} \sum_{\beta}^{N}\left\{\left|\phi_{\alpha}^{i}(\mathbf{r} \eta)\right|^{2}\left|\phi_{\beta}^{i}\left(\mathbf{r}^{\prime} \eta^{\prime}\right)\right|^{2}\right. \\
& \left.-\phi_{\alpha}^{i *}(\mathbf{r} \eta) \phi_{\beta}^{i *}\left(\mathbf{r}^{\prime} \eta^{\prime}\right) \phi_{\beta}^{i}(\mathbf{r} \eta) \phi_{\alpha}^{i}\left(\mathbf{r}^{\prime} \eta^{\prime}\right)\right\}, \\
& \Gamma_{i_{1} j_{1}}^{i j}\left(\mathbf{r} \mathbf{r}^{\prime}\right)=\iint d \eta d \eta^{\prime} \sum_{\beta}^{N-1}(-1)^{\bar{n}_{i_{1}}+\bar{n}_{\beta}\left(i_{1}\right)}(-1)^{\bar{n}_{j_{1}}+\bar{n}_{\beta}\left(j_{1}\right)} \\
& \times\left\{\phi_{i_{1}}^{i *}(\mathbf{r} \eta) \phi_{\beta}^{i *}\left(\mathbf{r}^{\prime} \eta^{\prime}\right) \phi_{j_{1}}^{j}(\mathbf{r} \eta) \phi_{\beta}^{j}\left(\mathbf{r}^{\prime} \eta^{\prime}\right)\right. \\
& -\phi_{i_{1}}^{i *}(\mathbf{r} \eta) \phi_{\beta}^{i *}\left(\mathbf{r}^{\prime} \eta^{\prime}\right) \phi_{\beta}^{j}(\mathbf{r} \eta) \phi_{j_{1}}^{j}\left(\mathbf{r}^{\prime} \eta^{\prime}\right) \\
& -\phi_{\beta}^{i *}(\mathbf{r} \eta) \phi_{i_{1}}^{i *}\left(\mathbf{r}^{\prime} \eta^{\prime}\right) \phi_{j_{1}}^{j}(\mathbf{r} \eta) \phi_{\beta}^{j}\left(\mathbf{r}^{\prime} \eta^{\prime}\right) \\
& \left.+\phi_{\beta}^{i *}(\mathbf{r} \eta) \phi_{i_{1}}^{i *}\left(\mathbf{r}^{\prime} \eta^{\prime}\right) \phi_{\beta}^{j}(\mathbf{r} \eta) \phi_{j_{1}}^{j}\left(\mathbf{r}^{\prime} \eta^{\prime}\right)\right\},
\end{aligned}
$$

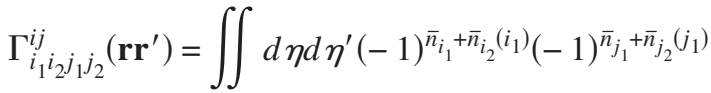

$$
\begin{aligned}
& \times\left\{\phi_{i_{1}}^{i *}(\mathbf{r} \eta) \phi_{i_{2}}^{i *}\left(\mathbf{r}^{\prime} \eta^{\prime}\right) \phi_{j_{1}}^{j}(\mathbf{r} \eta) \phi_{j_{2}}^{j}\left(\mathbf{r}^{\prime} \eta^{\prime}\right)\right. \\
& -\phi_{i_{1}}^{i *}(\mathbf{r} \eta) \phi_{i_{2}}^{i *}\left(\mathbf{r}^{\prime} \eta^{\prime}\right) \phi_{j_{2}}^{j}(\mathbf{r} \eta) \phi_{j_{1}}^{j}\left(\mathbf{r}^{\prime} \eta^{\prime}\right) \\
& -\phi_{i_{2}}^{i *}(\mathbf{r} \eta) \phi_{i_{1}}^{i *}\left(\mathbf{r}^{\prime} \eta^{\prime}\right) \phi_{j_{1}}^{j}(\mathbf{r} \eta) \phi_{j_{2}}^{j}\left(\mathbf{r}^{\prime} \eta^{\prime}\right) \\
& \left.+\phi_{i_{2}}^{i *}(\mathbf{r} \eta) \phi_{i_{1}}^{i *}\left(\mathbf{r}^{\prime} \eta^{\prime}\right) \phi_{j_{2}}^{j}(\mathbf{r} \eta) \phi_{j_{1}}^{j}\left(\mathbf{r}^{\prime} \eta^{\prime}\right)\right\} \text {, }
\end{aligned}
$$


where $\bar{n}_{i_{1}}$ is the number of functions which precede $\phi_{i_{1}}^{i}(\mathbf{r} \eta)$ in the SD and $\bar{n}_{i_{1}}\left(i_{2}\right)$ is the number of functions which precede $\phi_{i_{1}}^{i}(\mathbf{r} \eta)$ after annihilating $\phi_{i_{2}}^{i}(\mathbf{r} \eta)$. The summation in the second term of Eq. (4) is taken over the states, $\Phi_{i}$ and $\Phi_{j}$, which are different from each other in one constituent singleparticle wave function. The summation in the third term of Eq. (4) is over states, $\Phi_{i}$ and $\Phi_{j}$, which are different from each other in two constituent ones. The correct ground-state PD can, in principle, be searched by utilizing the infinite number of the SDs.

Here note that the PD is kept $N$-representable so far as the PD is varied within a form of Eq. (4), even though the finite number of SDs are used in the expansion of Eq. (1). This is a strong merit of the present scheme because the sum in Eq. (1) is compelled to be truncated to a finite number in actual calculations.

\section{B. Effective equation for the ground-state PD}

In this section, we shall derive the effective equation for the ground-state PD by means of the density functional approach. The universal functional of the PD functional theory is defined as $6,7,12,13,20$

$$
\begin{aligned}
F\left[\gamma^{(2)}\right] & =\operatorname{Min}_{\Psi \rightarrow \gamma^{(2)}}\langle\Psi|\hat{T}+\hat{W}| \Psi\rangle \\
& =\left\langle\Psi\left[\gamma^{(2)}\right]|\hat{T}+\hat{W}| \Psi\left[\gamma^{(2)}\right]\right\rangle,
\end{aligned}
$$

where $\hat{T}$ and $\hat{W}$ are operators of the kinetic energy and electron-electron interaction, respectively. The right-hand side of the first line of Eq. (8) denotes the searching of the minimum for the expectation value of $\hat{T}+\hat{W}$ over all antisymmetric wave functions that yield a prescribed $\gamma^{(2)}\left(\mathbf{r} \mathbf{r}^{\prime} ; \mathbf{r} \mathbf{r}^{\prime}\right)$, and $\Psi\left[\gamma^{(2)}\right]$ which appears in the second line is the minimizing wave function. Using Eq. (8), the Hohenberg-Kohn theorems for the PD functional theory can be proved $6,7,12,13,20$ in a similar way to the constrained-search theory. ${ }^{52-60}$ According to the variational principle with respect to the PD (the second theorem), the ground-state PD gives the minimum of the following total energy functional:

$$
\begin{aligned}
E_{v_{\text {ext }}\left[\gamma^{(2)}\right]=} & F\left[\gamma^{(2)}\right] \\
& +\iint \frac{v_{\text {ext }}(\mathbf{r})+v_{\text {ext }}\left(\mathbf{r}^{\prime}\right)}{N-1} \gamma^{(2)}\left(\mathbf{r} \mathbf{r}^{\prime} ; \mathbf{r r}^{\prime}\right) d^{3} r d^{3} r^{\prime} \\
= & T\left[\gamma^{(2)}\right]+e^{2} \iint \frac{\gamma^{(2)}\left(\mathbf{r r}^{\prime} ; \mathbf{r} \mathbf{r}^{\prime}\right)}{\left|\mathbf{r}-\mathbf{r}^{\prime}\right|} d^{3} r d^{3} r^{\prime} \\
& +\iint \frac{v_{\text {ext }}(\mathbf{r})+v_{\text {ext }}\left(\mathbf{r}^{\prime}\right)}{N-1} \gamma^{(2)}\left(\mathbf{r} \mathbf{r}^{\prime} ; \mathbf{r r}^{\prime}\right) d^{3} r d^{3} r^{\prime}
\end{aligned}
$$

with

$$
T\left[\gamma^{(2)}\right]=\left\langle\Psi\left[\gamma^{(2)}\right]|\hat{T}| \Psi\left[\gamma^{(2)}\right]\right\rangle,
$$

where $v_{\text {ext }}(\mathbf{r})$ is the external potential.

The variational principle has to be performed with varying the PD within the set of the $N$-representable PDs. In the present scheme, the coefficients $\left\{C_{i}\right\}$ are varied with singleparticle wave functions fixed. To guarantee Eq. (4) to be $N$-representable, we have to find the condition for meeting the requirement that Eq. (1) is the antisymmetric wave function. Since $\Phi_{i}$ in Eq. (1) are determinantal wave functions and orthonormal to each other, such a condition is given by

$$
\sum_{i}\left|C_{i}\right|^{2}=1
$$

It can be easily shown that the condition (11) is equivalent to

$$
\iint \gamma^{(2)}\left(\mathbf{r} \mathbf{r}^{\prime} ; \mathbf{r} \mathbf{r}^{\prime}\right) d^{3} r d^{3} r^{\prime}=\frac{N(N-1)}{2}
$$

under the assumption that $\gamma^{(2)}\left(\mathbf{r r}^{\prime} ; \mathbf{r r}^{\prime}\right)$ is given by Eq. (4).

Let us consider the minimization of Eq. (9) under the condition of Eq. (12). We have only to take the variation of the following functional with respect to the PD:

$$
\begin{aligned}
\Omega_{v_{e x t}\left[\gamma^{(2)}\right]=} & E_{v_{e x t}\left[\gamma^{(2)}\right]-\mu\left\{\frac{2}{N(N-1)}\right.} \\
& \left.\times \iint \gamma^{(2)}\left(\mathbf{r} \mathbf{r}^{\prime} ; \mathbf{r r}^{\prime}\right) d^{3} r d^{3} r^{\prime}-1\right\},
\end{aligned}
$$

where $\mu$ is the Lagrange multiplier for the condition of Eq. (12). As mentioned above, since $\gamma_{\mathrm{SSD}, i}^{(2)}\left(\mathbf{r r}^{\prime} ; \mathbf{r r}^{\prime}\right), \Gamma_{i_{1} j_{1}}^{i j}\left(\mathbf{r r}^{\prime}\right)$, and $\Gamma_{i_{1} i_{2} j_{1} j_{2}}^{i j}\left(\mathbf{r} \mathbf{r}^{\prime}\right)$ are given functions, the PD can be written as $\gamma^{(2)}\left[\left\{C_{i}\right\}\right]\left(\mathbf{r} \mathbf{r}^{\prime} ; \mathbf{r r}^{\prime}\right)$. Therefore, the variation of Eq. (13) with respect to the PD is written by

$$
\delta \Omega_{v_{e x t}}\left[\gamma^{(2)}\right]=\sum_{i} \frac{\delta \Omega_{v_{e x t}}}{\delta C_{i}^{*}} \delta C_{i}^{*}+\sum_{i} \frac{\delta \Omega_{v_{e x t}}}{\delta C_{i}} \delta C_{i} .
$$

Since $C_{i}^{*}$ and $C_{i}$ are independent of each other, $\delta \Omega_{v_{e x t}}\left[\gamma^{(2)}\right]$ $=0$ yields

$$
\frac{\delta \Omega_{v_{e x t}}}{\delta C_{i}^{*}}=0
$$

and

$$
\frac{\delta \Omega_{v_{e x t}}}{\delta C_{i}}=0
$$

Both Eqs. (15) and (16) give an equivalent equation as follows: 


$$
\begin{aligned}
\mu C_{i}^{0}= & \left.\frac{\delta T\left[\gamma^{(2)}\left[\left\{C_{i}\right\}\right]\right]}{\delta C_{i}^{*}}\right|_{\left\{C_{i}\right\}=\left\{C_{i}^{0}\right\}}+C_{i}^{0} \iint\left[\frac{e^{2}}{\left|\mathbf{r}-\mathbf{r}^{\prime}\right|}+\frac{1}{N-1}\left\{v_{e x t}(\mathbf{r})+v_{e x t}\left(\mathbf{r}^{\prime}\right)\right\}\right] \gamma_{\mathrm{SSD}, i}^{(2)}\left(\mathbf{r r}^{\prime} ; \mathbf{r r}^{\prime}\right) d^{3} r d^{3} r^{\prime} \\
& +\sum_{j}{ }^{\prime} \frac{C_{j}^{0}}{2} \iint\left[\frac{e^{2}}{\left|\mathbf{r}-\mathbf{r}^{\prime}\right|}+\frac{1}{N-1}\left\{v_{e x t}(\mathbf{r})+v_{e x t}\left(\mathbf{r}^{\prime}\right)\right\}\right] \Gamma_{i_{1} j_{1}}^{i j}\left(\mathbf{r} \mathbf{r}^{\prime}\right) d^{3} r d^{3} r^{\prime} \\
& +\sum_{j}{ }^{\prime \prime} \frac{C_{j}^{0}}{2} \iint\left[\frac{e^{2}}{\left|\mathbf{r}-\mathbf{r}^{\prime}\right|}+\frac{1}{N-1}\left\{v_{\text {ext }}(\mathbf{r})+v_{e x t}\left(\mathbf{r}^{\prime}\right)\right\}\right] \Gamma_{i_{1} i_{2} j_{1} j_{2}}^{i j}\left(\mathbf{r} \mathbf{r}^{\prime}\right) d^{3} r d^{3} r^{\prime}
\end{aligned}
$$

This is the effective equation of the present scheme. Solving Eq. (17), and substituting the solutions $\left\{C_{i}^{0}\right\}$ into Eq. (4), we can, in principle, get the ground-state PD.

The efficiency of the actual calculation is dependent on the choice of the set of single-particle wave functions. In this paper, eigenfunctions of the effective initial scheme $e^{22,23}$ are chosen in hope of including the correlation effects more efficiently with fewer number of SDs of Eq. (1). Details of the calculations are shown in Sec. V.

\section{RESTRICTIVE CONDITIONS ON THE KINETIC ENERGY FUNCTIONAL}

Approximate forms of the kinetic energy functional are indispensable for the PD functional theory. One of useful and sound methods to develop such approximate functionals is to utilize exact relations and bounds of the energy functional as restrictive conditions on its approximate forms. ${ }^{44-51}$ In this section, we shall derive two restrictive conditions on approximate forms of $T\left[\gamma^{(2)}\right]$. One is derived in Sec. III A by considering the physical meaning of the Lagrange multiplier $\mu$ of Eq. (17) and the other is derived from the scaling property of $T\left[\gamma^{(2)}\right]$ in Sec. III B.

\section{A. Meaning of the Lagrange multiplier}

It is shown in Sec. II that the variational problem with respect to the PD can be reformulated into solving a minimization problem for the functional $\Omega_{v_{e x t}}\left[\gamma^{(2)}\right]$. Since the wave function $\Psi\left[\gamma^{(2)}\right]$ yields $\gamma^{(2)}\left(\mathbf{r r}^{\prime} ; \mathbf{r r}^{\prime}\right)$ and minimizes the expectation value of $\hat{T}+\hat{W}$, Eq. (13) is rewritten as

$$
\begin{aligned}
\Omega_{v_{\text {ext }}\left[\gamma^{(2)}\right]=} & \int \Psi^{*}\left[\gamma^{(2)}\right] \hat{H}_{v_{\text {ext }}} \Psi\left[\gamma^{(2)}\right] d \tau \\
& -\mu\left\{\int \Psi^{*}\left[\gamma^{(2)}\right] \Psi\left[\gamma^{(2)}\right] d \tau-1\right\},
\end{aligned}
$$

where $d \tau$ is a volume element in the configuration space of the system and $\hat{H}_{v_{\text {ext }}}$ is the Hamiltonian of the system. Therefore, using the chain rule for the functional derivative, $\delta \Omega_{v_{e x t}}$ is written as

$$
\begin{aligned}
\delta \Omega_{v_{e x t}}= & \int \frac{\delta \Omega_{v_{e x t}}}{\delta \Psi\left[\gamma^{(2)}\right]} \frac{\delta \Psi\left[\gamma^{(2)}\right]}{\delta \gamma^{(2)}\left(\mathbf{r r}^{\prime}, \mathbf{r r}^{\prime}\right)} \delta \gamma^{(2)}\left(\mathbf{r r}^{\prime}, \mathbf{r r}^{\prime}\right) d^{3} r d^{3} r^{\prime} d \tau \\
& +\int \frac{\delta \Omega_{v_{e x t}}}{\delta \Psi^{*}\left[\gamma^{(2)}\right]} \frac{\delta \Psi^{*}\left[\gamma^{(2)}\right]}{\delta \gamma^{(2)}\left(\mathbf{r r}^{\prime}, \mathbf{r} \mathbf{r}^{\prime}\right)} \\
& \times \delta \gamma^{(2)}\left(\mathbf{r} \mathbf{r}^{\prime}, \mathbf{r r}^{\prime}\right) d^{3} r d^{3} r^{\prime} d \tau .
\end{aligned}
$$

Substituting Eq. (18) into Eq. (19), we have

$$
\begin{aligned}
\delta \Omega_{v_{\text {ext }}}= & \int\left\{\Psi^{*}\left[\gamma^{(2)}\right] \hat{H}_{v_{\text {ext }}}-\mu \Psi^{*}\left[\gamma^{(2)}\right]\right\} \delta \Psi d \tau \\
& +\int\left\{\hat{H}_{v_{\text {ext }}} \Psi\left[\gamma^{(2)}\right]-\mu \Psi\left[\gamma^{(2)}\right]\right\} \delta \Psi^{*} d \tau .
\end{aligned}
$$

According to the variational principle with respect to the PD (the second theorem), $\delta \Omega_{v_{\text {ext }}}$ becomes zero when the PD is equal to the ground-state PD. If we denote the ground-state PD by $\gamma_{0}^{(2)}\left(\mathbf{r r}^{\prime}, \mathbf{r r}^{\prime}\right)$, then the minimum condition leads to

$$
\hat{H}_{v_{\text {ext }}} \Psi\left[\gamma_{0}^{(2)}\right]=\mu \Psi\left[\gamma_{0}^{(2)}\right] .
$$

Furthermore, according to the one-to-one correspondence between the ground-state PD and the ground-state wave function (the first theorem), $\Psi\left[\gamma_{0}^{(2)}\right]$ is equal to the ground-state wave function $\Psi_{0}$. Therefore, we finally get

$$
\hat{H}_{v_{e x t}} \Psi_{0}=\mu \Psi_{0} .
$$

Equation (22) immediately means that

$$
\mu=\text { ground-state energy. }
$$

The physical meaning of $\mu$, i.e., Eq. (23), can be used as a restrictive condition on the approximate form of $T\left[\gamma^{(2)}\right]$. Multiplying $C_{i}^{0 *}$ on both sides of the effective Eq. (17), and summing over $i$, then we obtain

$$
\begin{aligned}
\mu= & \left.\sum_{i} C_{i}^{0 *} \frac{\delta T\left[\gamma^{(2)}\left[\left\{C_{i}\right\}\right]\right]}{\delta C_{i}^{*}}\right|_{\left\{C_{i}\right\}=\left\{C_{i}^{0}\right\}} \\
& +e^{2} \iint \frac{\gamma_{0}^{(2)}\left(\mathbf{r r}^{\prime} ; \mathbf{r r}^{\prime}\right)}{\left|\mathbf{r}-\mathbf{r}^{\prime}\right|} d^{3} r d^{3} r^{\prime} \\
& +\frac{1}{N-1} \iint\left\{v_{e x t}(\mathbf{r})+v_{e x t}\left(\mathbf{r}^{\prime}\right)\right\} \gamma_{0}^{(2)}\left(\mathbf{r r}^{\prime} ; \mathbf{r r}^{\prime}\right) d^{3} r d^{3} r^{\prime},
\end{aligned}
$$


where we use Eq. (11). On the other hand, since $\mu$ is equal to the ground-state energy, we have

$$
\begin{aligned}
\mu= & T\left[\gamma_{0}^{(2)}\right]+e^{2} \iint \frac{\gamma_{0}^{(2)}\left(\mathbf{r} \mathbf{r}^{\prime} ; \mathbf{r r}^{\prime}\right)}{\left|\mathbf{r}-\mathbf{r}^{\prime}\right|} d^{3} r d^{3} r^{\prime} \\
& +\frac{1}{N-1} \iint\left\{v_{e x t}(\mathbf{r})+v_{e x t}\left(\mathbf{r}^{\prime}\right)\right\} \gamma_{0}^{(2)}\left(\mathbf{r} \mathbf{r}^{\prime} ; \mathbf{r r}^{\prime}\right) d^{3} r d^{3} r^{\prime}
\end{aligned}
$$

Comparing Eq. (24) with Eq. (25), we get

$$
\left.\sum_{i} C_{i}^{0 *} \frac{\delta T\left[\gamma^{(2)}\left[\left\{C_{i}\right\}\right]\right]}{\delta C_{i}^{*}}\right|_{\left\{C_{i}\right\}=\left\{C_{i}^{0}\right\}}=T\left[\gamma_{0}^{(2)}\right] .
$$

Using Eq. (4) and the chain rule for functional derivatives, the left-hand side of Eq. (26) can be rewritten by

$$
\left.\iint \gamma_{0}^{(2)}\left(\mathbf{r r}^{\prime} ; \mathbf{r r}^{\prime}\right) \frac{\delta T\left[\gamma^{(2)}\left[\left\{C_{i}\right\}\right]\right]}{\delta \gamma^{(2)}\left[\left\{C_{i}\right\}\right]\left(\mathbf{r r}^{\prime} ; \mathbf{r r}^{\prime}\right)}\right|_{\gamma^{(2)}\left[\left\{C_{i}\right\}\right]=\gamma_{0}^{(2)}} d^{3} r d^{3} r^{\prime}
$$

Thus, we obtain

$$
\begin{aligned}
& \left.\iint \gamma_{0}^{(2)}\left(\mathbf{r r}^{\prime} ; \mathbf{r r}^{\prime}\right) \frac{\delta T\left[\gamma^{(2)}\left[\left\{C_{i}\right\}\right]\right]}{\delta \gamma^{(2)}\left[\left\{C_{i}\right\}\right]\left(\mathbf{r r}^{\prime} ; \mathbf{r r}^{\prime}\right)}\right|_{\gamma^{(2)}\left[\left\{C_{i}\right\}\right]=\gamma_{0}^{(2)}} d^{3} r d^{3} r^{\prime} \\
& \quad=T\left[\gamma_{0}^{(2)}\right] .
\end{aligned}
$$

Note that the functional derivative in the left-hand side of Eq. (27) should be calculated by varying the PD within a form of Eq. (4).

Equation (26) or (27) can be a restrictive condition on approximate forms of $T\left[\gamma^{(2)}\right]$. For example, if $T\left[\gamma^{(2)}\right]$ is supposed to be expressed by the integral of a power series of $\gamma^{(2)}\left(\mathbf{r} \mathbf{r}^{\prime} ; \mathbf{r r}^{\prime}\right)$, it is shown from Eq. (26) or (27) that the coefficients of the power series should be zero except for the linear term. Thus, Eq. (26) or (27) can be effectively used in developing approximate forms of $T\left[\gamma^{(2)}\right]$.

\section{B. Scaling property of the kinetic energy functional}

Let us derive the other restrictive condition on $T\left[\gamma^{(2)}\right]$. The scaling property of the kinetic energy functional $T\left[\gamma^{(2)}\right]$ is given by ${ }^{10,20,22}$

$$
T\left[\gamma_{\zeta}^{(2)}\right]=\zeta^{2} T\left[\gamma^{(2)}\right],
$$

where $\gamma_{\zeta}^{(2)}\left(\mathbf{r} \mathbf{r}^{\prime} ; \mathbf{r} \mathbf{r}^{\prime}\right)$ denotes the scaled PD that is defined as $\zeta^{6} \gamma^{(2)}\left(\zeta \mathbf{r} \zeta \mathbf{r}^{\prime} ; \zeta \mathbf{r} \zeta \mathbf{r}^{\prime}\right){ }^{10,20,22}$ Applying $\lim _{\zeta \rightarrow 1} \frac{\partial}{\partial \zeta}$ to Eq. (28) on both sides, we obtain

$$
\begin{aligned}
2 T\left[\gamma^{(2)}\right]= & \iint \frac{\delta T\left[\gamma^{(2)}\right]}{\delta \gamma^{(2)}\left(\mathbf{r r}^{\prime} ; \mathbf{r r}^{\prime}\right)}\left\{6 \gamma^{(2)}\left(\mathbf{r r}^{\prime} ; \mathbf{r r}^{\prime}\right)\right. \\
& \left.+\mathbf{r} \cdot \nabla \gamma^{(2)}\left(\mathbf{r r}^{\prime} ; \mathbf{r r}^{\prime}\right)+\mathbf{r}^{\prime} \cdot \nabla^{\prime} \gamma^{(2)}\left(\mathbf{r r}^{\prime} ; \mathbf{r} \mathbf{r}^{\prime}\right)\right\} d^{3} r d^{3} r^{\prime},
\end{aligned}
$$

where $\nabla$ and $\nabla^{\prime}$ denote the nabla operators with respect to $\mathbf{r}$ and $\mathbf{r}^{\prime}$, respectively. Using the fact that integrals over an infinitely distant surface vanish in isolated systems, Eq. (29) is rewritten as

$$
\begin{aligned}
2 T\left[\gamma^{(2)}\right]= & -\iint \mathbf{r} \cdot\left\{\nabla \frac{\delta T\left[\gamma^{(2)}\right]}{\delta \gamma^{(2)}\left(\mathbf{r r}^{\prime} ; \mathbf{r r}^{\prime}\right)}\right\} \gamma^{(2)}\left(\mathbf{r r}^{\prime} ; \mathbf{r r}^{\prime}\right) d^{3} r d^{3} r^{\prime} \\
& -\iint \mathbf{r}^{\prime} \cdot\left\{\nabla^{\prime} \frac{\delta T\left[\gamma^{(2)}\right]}{\delta \gamma^{(2)}\left(\mathbf{r r}^{\prime} ; \mathbf{r r}^{\prime}\right)}\right\} \\
& \times \gamma^{(2)}\left(\mathbf{r} \mathbf{r}^{\prime} ; \mathbf{r r}^{\prime}\right) d^{3} r d^{3} r^{\prime} .
\end{aligned}
$$

Approximate forms that satisfy Eq. (30) are regarded as favorable ones in a sense that they are consistent with the scaling property of $T\left[\gamma^{(2)}\right]$. Equation (30) is the second restrictive condition on the approximate form of $T\left[\gamma^{(2)}\right]$.

Thus, two restrictive conditions on approximate forms of $T\left[\gamma^{(2)}\right]$ are obtained, i.e., Eqs. (26) [or (27)] and (30). These are actually used in the subsequent section for the development of approximate functionals.

\section{PROPOSAL OF APPROXIMATE FORMS OF THE KINETIC ENERGY FUNCTIONAL}

In this section, using two restrictive conditions derived in Sec. III, we propose approximate forms of $T\left[\gamma^{(2)}\right]$. In order to make approximate forms satisfy the first restrictive condition of Eq. (26), we shall start with the following expression:

$$
T\left[\gamma^{(2)}\right]=\iint f\left(\mathbf{r}, \mathbf{r}^{\prime}\right) \gamma^{(2)}\left(\mathbf{r} \mathbf{r}^{\prime} ; \mathbf{r} \mathbf{r}^{\prime}\right) d^{3} r d^{3} r^{\prime},
$$

where $f\left(\mathbf{r}, \mathbf{r}^{\prime}\right)$ is an arbitrary function of $\mathbf{r}$ and $\mathbf{r}^{\prime}$. By using Eq. (4), it can easily be confirmed that Eq. (31) fulfills Eq. (26). Namely, Eq. (31) is consistent with the first restrictive condition on $T\left[\gamma^{(2)}\right]$. The explicit form of $f\left(\mathbf{r}, \mathbf{r}^{\prime}\right)$ is determined by the second restrictive condition of Eq. (30). Substituting Eq. (31) into Eq. (30), we get

$$
\begin{gathered}
\iint\left\{2 f\left(\mathbf{r}, \mathbf{r}^{\prime}\right)+\mathbf{r} \cdot \nabla f\left(\mathbf{r}, \mathbf{r}^{\prime}\right)+\mathbf{r}^{\prime} \cdot \nabla^{\prime} f\left(\mathbf{r}, \mathbf{r}^{\prime}\right)\right\} \\
\quad \times \gamma^{(2)}\left(\mathbf{r r}^{\prime} ; \mathbf{r} \mathbf{r}^{\prime}\right) d^{3} r d^{3} r^{\prime}=0 .
\end{gathered}
$$

As the sufficient condition for satisfying Eq. (32), the differential equation for $f\left(\mathbf{r}, \mathbf{r}^{\prime}\right)$ is obtained

$$
2 f\left(\mathbf{r}, \mathbf{r}^{\prime}\right)+\mathbf{r} \cdot \nabla f\left(\mathbf{r}, \mathbf{r}^{\prime}\right)+\mathbf{r}^{\prime} \cdot \nabla^{\prime} f\left(\mathbf{r}, \mathbf{r}^{\prime}\right)=0 .
$$

We can find various solutions for this differential equation and some of these are

$$
\begin{gathered}
f_{1}\left(\mathbf{r}, \mathbf{r}^{\prime}\right)=\frac{1}{\left|\mathbf{r}-\mathbf{r}^{\prime}\right|^{2}}, \\
f_{2}\left(\mathbf{r}, \mathbf{r}^{\prime}\right)=\frac{1}{r^{2}}+\frac{1}{r^{\prime 2}}, \\
f_{3}\left(\mathbf{r}, \mathbf{r}^{\prime}\right)=\frac{1}{r r^{\prime}}, \\
f_{4}\left(\mathbf{r}, \mathbf{r}^{\prime}\right)=\frac{1}{\left|\mathbf{r}-\mathbf{r}^{\prime}\right|}\left(\frac{1}{r}+\frac{1}{r^{\prime}}\right), \\
f_{5}\left(\mathbf{r}, \mathbf{r}^{\prime}\right)=\frac{1}{\left|\mathbf{r}+\mathbf{r}^{\prime}\right|^{2}},
\end{gathered}
$$




$$
\begin{gathered}
f_{6}\left(\mathbf{r}, \mathbf{r}^{\prime}\right)=\frac{1}{\left|\mathbf{r}+\mathbf{r}^{\prime}\right|}\left(\frac{1}{r}+\frac{1}{r^{\prime}}\right), \\
f_{7}\left(\mathbf{r}, \mathbf{r}^{\prime}\right)=\frac{1}{\sqrt{r r^{\prime}}}\left(\frac{1}{r}+\frac{1}{r^{\prime}}\right) .
\end{gathered}
$$

If we choose an arbitrary linear combination of Eqs. (34)-(40) as $f\left(\mathbf{r}, \mathbf{r}^{\prime}\right)$, then Eq. (33) holds. In this paper, we adopt as $f\left(\mathbf{r}, \mathbf{r}^{\prime}\right)$ a linear combination of two functions that are chosen from Eqs. (34)-(40). Namely, the approximate form of $T\left[\gamma^{(2)}\right]$, which is used in the present scheme, is given by

$$
\begin{aligned}
& T\left[\gamma^{(2)}\right]= \iint\left\{K f_{\mu}\left(\mathbf{r}, \mathbf{r}^{\prime}\right)+K^{\prime} f_{\nu}\left(\mathbf{r}, \mathbf{r}^{\prime}\right)\right\} \\
& \times \gamma^{(2)}\left(\mathbf{r} \mathbf{r}^{\prime} ; \mathbf{r r}^{\prime}\right) d^{3} r d^{3} r^{\prime} \\
&(\mu, \nu=1,2, \ldots, 7, \mu<\nu),
\end{aligned}
$$

where $K$ and $K^{\prime}$ are arbitrary constants. It should be stressed that Eq. (41) is the favorable functional that satisfies two restrictive conditions, independently of the values of $K$ and $K^{\prime}$.

\section{EVALUATION OF THE PRESENT SCHEME INCLUDING APPROXIMATE FORMS OF THE KINETIC ENERGY FUNCTIONAL}

In order to evaluate the abilities and limitations of the present scheme including approximate forms [Eq. (41)], test calculations for the electronic structure of a neutral neon atom are performed. In Sec. V A, we shall first explain how to evaluate the present scheme. In Sec. V B, we mention the details of numerical calculations such as a calculation procedure and expressions used here. The results and discussions are given in Sec. V C. The position of the present scheme in the field of the PD functional theory is argued in Sec. V D.

\section{A. Evaluation method}

In order to evaluate the abilities and limitations of the present scheme including Eq. (41), we determine the values of $K$ and $K^{\prime}$ as follows. The procedure is illustrated in a part of Fig. 1. For a fixed value of $K$, the value of $K^{\prime}$ is determined by requiring that the root-mean-square error of the electron density $(\Delta \rho)$ is minimized with respect to $K^{\prime}$. As often as the value of $K$ is changed, the value of $K^{\prime}$, which minimizes $\Delta \rho$, is obtained similarly. Namely, the set of pairs $\left(K, K^{\prime}\right)$ is obtained. Among the set of pairs, we choose a pair $\left(K, K^{\prime}\right)$ which reproduces the total energy $\left(E_{\text {total }}\right)$ with enough accuracy. As the reference data of the electron density and the total energy, we utilize results of the configuration interaction $(\mathrm{CI})$ method. ${ }^{61,62}$

If $\Delta \rho$ becomes a small value, and if $E_{\text {total }}$ approaches to the result of the CI method, then the resultant PD seems to be close to the ground-state PD. Therefore, we evaluate the abilities and limitations of the present scheme including Eq. (41) by investigating to what extent the electron density and the total energy are reproduced.

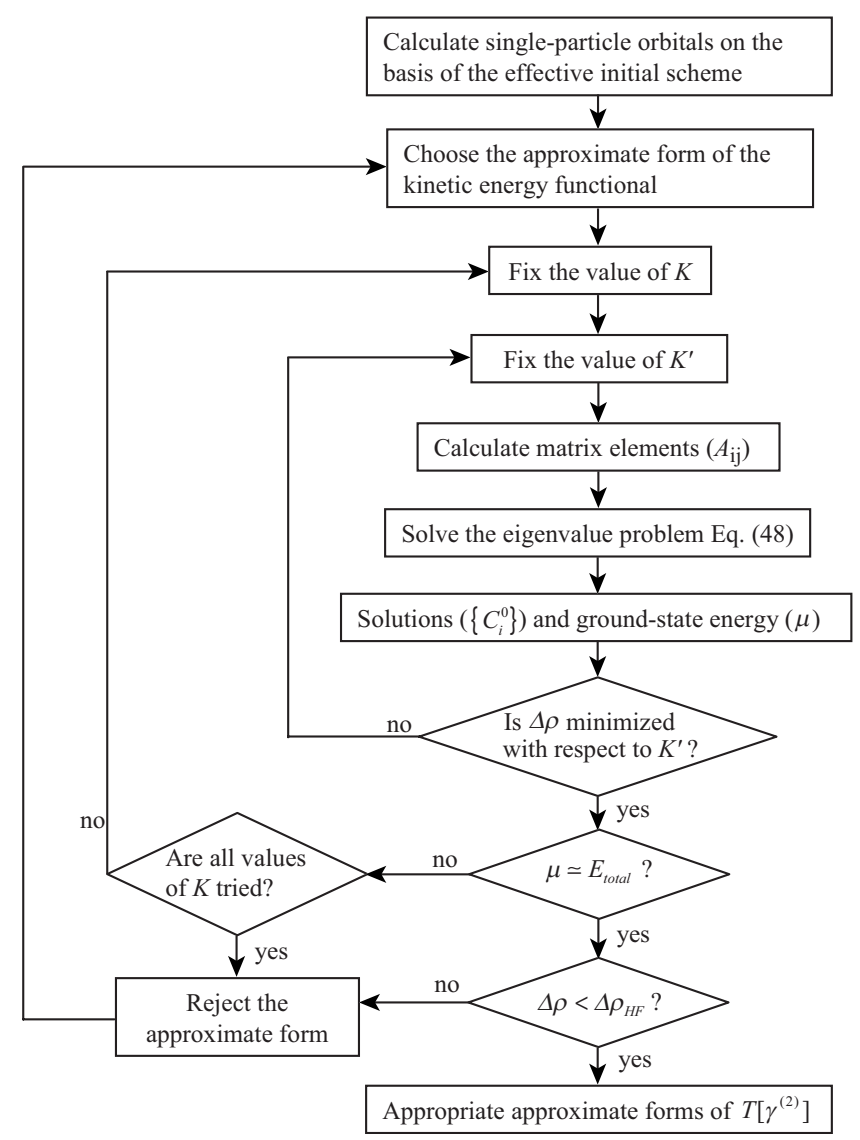

FIG. 1. Flowchart of numerical calculations.

If the total energy cannot be reproduced, and/or if $\Delta \rho$ is larger than that of the Hartree-Fock approximation $\left(\Delta \rho_{H F}\right.$ $=0.11892$ ), then we remove such approximate functionals from candidates of appropriate ones of the present scheme. Along this evaluation method, we test a total of 21 functionals shown in Eq. (41). Further discussion will be done in the forthcoming Sec. V C.

As appropriate ones, we finally obtain the following six approximate forms

(i) $\iint\left\{K\left(\frac{1}{r^{2}}+\frac{1}{r^{\prime 2}}\right)+\frac{K^{\prime}}{r r^{\prime}}\right\} \gamma^{(2)}\left(\mathbf{r r}^{\prime} ; \mathbf{r r}^{\prime}\right) d^{3} r d^{3} r^{\prime}$,

(ii) $\iint\left\{\frac{K}{r r^{\prime}}+\frac{K^{\prime}}{\left|\mathbf{r}+\mathbf{r}^{\prime}\right|^{2}}\right\} \gamma^{(2)}\left(\mathbf{r} \mathbf{r}^{\prime} ; \mathbf{r} \mathbf{r}^{\prime}\right) d^{3} r d^{3} r^{\prime}$,

(iii) $\iint\left(\frac{K}{\left|\mathbf{r}-\mathbf{r}^{\prime}\right|}+\frac{K^{\prime}}{\left|\mathbf{r}+\mathbf{r}^{\prime}\right|}\right)\left(\frac{1}{r}+\frac{1}{r^{\prime}}\right) \gamma^{(2)}\left(\mathbf{r} \mathbf{r}^{\prime} ; \mathbf{r r}^{\prime}\right) d^{3} r d^{3} r^{\prime}$,

(iv) $\iint\left\{\frac{K}{\left|\mathbf{r}-\mathbf{r}^{\prime}\right|^{2}}+\frac{K^{\prime}}{\sqrt{r r^{\prime}}}\left(\frac{1}{r}+\frac{1}{r^{\prime}}\right)\right\} \gamma^{(2)}\left(\mathbf{r} \mathbf{r}^{\prime} ; \mathbf{r r}^{\prime}\right) d^{3} r d^{3} r^{\prime}$,

(v) $\iint\left\{\frac{K}{r r^{\prime}}+\frac{K^{\prime}}{\sqrt{r r^{\prime}}}\left(\frac{1}{r}+\frac{1}{r^{\prime}}\right)\right\} \gamma^{(2)}\left(\mathbf{r} \mathbf{r}^{\prime} ; \mathbf{r r}^{\prime}\right) d^{3} r d^{3} r^{\prime}$, 
(vi) $\iint\left\{\left(\frac{K}{\left|\mathbf{r}-\mathbf{r}^{\prime}\right|}+\frac{K^{\prime}}{\sqrt{r r^{\prime}}}\right)\left(\frac{1}{r}+\frac{1}{r^{\prime}}\right)\right\} \gamma^{(2)}\left(\mathbf{r} \mathbf{r}^{\prime} ; \mathbf{r r}^{\prime}\right) d^{3} r d^{3} r^{\prime}$.

The details of calculations will be mentioned in the subsequent Sec. V B.

\section{B. Calculation details}

As multiple SDs used in the expansion of Eq. (1), we choose the set of SDs which consist of the eigenfunctions of the effective initial scheme. ${ }^{22,23}$ Since the effective initial scheme can reproduce about $20 \%$ of the correlation energy for the neon atom, ${ }^{22,23}$ we may expect that the ground-state PD is expressed enough by fewer number of SDs. The eigenfunctions of the effective initial scheme, i.e., constituent single-particle orbitals of the SD of Eq. (1), are calculated on the basis of the usual spherical approximation (central-field approximation). ${ }^{22,63}$ The maximum values of the principal quantum number and azimuthal quantum number of orbitals are 48 and 47, respectively. A spherical hard wall with a large radius $\left(R_{0}\right)$ is introduced in calculating positive-energy states, similarly to other works where positive-energy states have been utilized in the perturbation calculation. ${ }^{64,65}$ Namely, radial wave functions vanish at the wall and are normalized in the sphere. In order to recover the state of a free atom as well as possible, we take 25 bohrs as the value of $R_{0}$ with reference to other works. ${ }^{64,65}$ Using orbitals thus obtained, we construct the ground-state SD and 460 singlyexcited SDs of the effective initial scheme. That is, a total of 461 SDs are used in test calculations.

Next, we shall rewrite the effective equations [Eq. (17)] for the purpose of numerical calculations. If we employ Eq. (41) as the approximate form of $T\left[\gamma^{(2)}\right]$, then Eq. (17) can be rewritten as

$$
\sum_{j} A_{i j} C_{j}^{0}=\mu C_{i}^{0}
$$

with

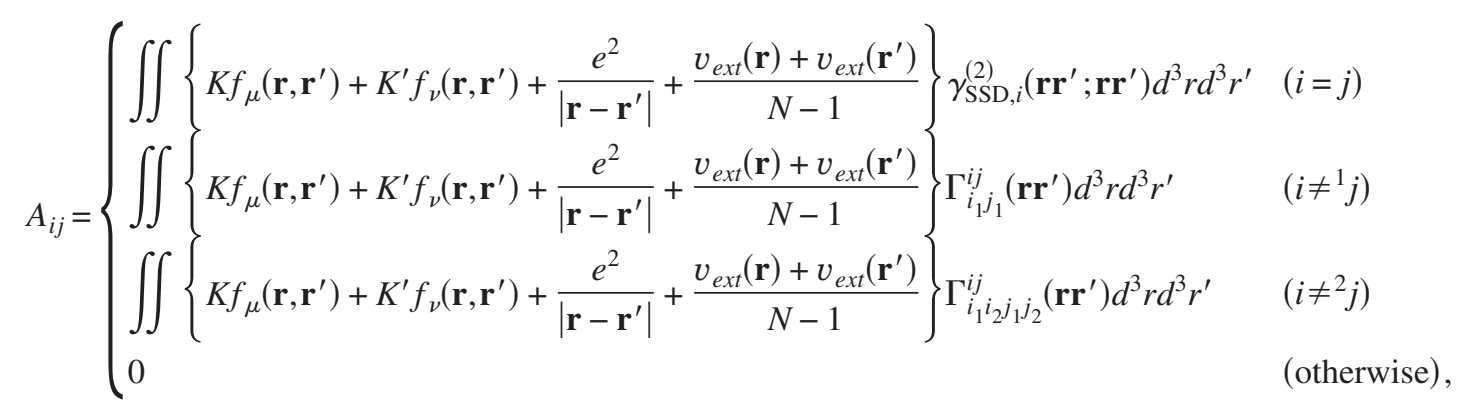

where the symbol $i \neq{ }^{1} j(i \neq 2 j)$ denotes that $\Phi_{j}$ is different from $\Phi_{i}$ by one (two) constituent orbital(s). Thus, the effective equation [Eq. (17)] is reduced to the eigenvalue problem with the Hamiltonian matrix $A$. In Appendix, the matrix elements $A_{i j}$ are rewritten in terms of single-particle orbitals that are calculated by the effective initial theory. Such expressions for $A_{i j}$ are used in actual calculations.

The flowchart of numerical calculations is shown in Fig. 1. In the calculations, we first perform the effective initial scheme so as to get the set of constituent orbitals of the SDs in Eq. (1). After choosing of the approximate form of $T\left[\gamma^{(2)}\right]$ and fixing the values of $K$ and $K^{\prime}$, we calculate the matrix elements $A_{i j}$ by using these orbitals (See, Appendix). Then, solving the eigenvalue problem Eq. (48), we obtain the solution $\left\{C_{i}^{0}\right\}$ and the ground-state energy $\mu$. These calculations are repeated for the other values of $K$ and $K^{\prime}$, though the way to determine their values has already been mentioned in Sec. V A.

TABLE I. The root-mean-square error of the electron density $(\Delta \rho)$, total energy $\left(E_{\text {total }}\right)$, and the error to the CI result $\left(E_{\mathrm{CI}}\right)$ for each approximate form. Values of $K$ and $K^{\prime}$, which are used in approximate forms, are

\begin{tabular}{|c|c|c|c|c|c|}
\hline & $K$ & $K^{\prime}$ & $\Delta \rho$ & $\begin{array}{c}E_{\text {total }} \\
(\mathrm{Ry})\end{array}$ & $\begin{array}{c}\frac{E_{\text {total }}-E_{\mathrm{CI}}}{E_{\mathrm{CI}}} \\
(\%)\end{array}$ \\
\hline (i) & 0.02360 & 0.47050 & 0.09250 & -257.8359 & -0.0078 \\
\hline (ii) & 1.783 & -2.4785 & 0.09397 & -257.8000 & -0.0217 \\
\hline (iii) & 2.601 & -1.9976 & 0.10787 & -257.8279 & -0.0109 \\
\hline (iv) & 0.1543 & 0.21627 & 0.09215 & -257.8705 & 0.0056 \\
\hline (v) & 0.2447 & 0.15650 & 0.09139 & -257.8547 & -0.0005 \\
\hline (vi) & 0.09267 & 0.19629 & 0.09198 & -257.8628 & 0.0026 \\
\hline
\end{tabular}
also shown. 
TABLE II. The ratio of the kinetic energy (potential energy) to the corresponding CI result for each approximate form is shown in the first column (second column). The virial ratio (VR) and the error to the value -2.0 are shown in the third and fourth columns, respectively.

\begin{tabular}{ccccc}
\hline \hline & $\begin{array}{c}\text { Kinetic energy } \\
(\%)\end{array}$ & $\begin{array}{c}\text { potential energy } \\
(\%)\end{array}$ & VR & $\begin{array}{c}-\frac{V R+2.0}{2.0} \\
(\%)\end{array}$ \\
\hline (i) & 95.588 & 97.790 & -2.0461 & 2.30 \\
(ii) & 95.601 & 97.790 & -2.0458 & 2.35 \\
(iii) & 95.582 & 97.786 & -2.0461 & 2.31 \\
(iv) & 95.578 & 97.792 & -2.0463 & 2.32 \\
(v) & 95.590 & 97.795 & -2.0461 & 2.31 \\
(vi) & 95.582 & 97.792 & -2.0463 & 2.31 \\
\hline \hline
\end{tabular}

\section{Results and discussions}

According to the evaluation method mentioned in Sec. $\mathrm{V}$ A, we find six appropriate functionals (i)-(vi) that are given in Eqs. (42)-(47). The values of $K, K^{\prime}, \Delta \rho$, and $E_{\text {total }}$ are listed in Table I. Judging from Table I, the approximate forms (i)-(vi) surely provide the correlated PDs in a sense that both the total energy and electron density are improved compared with those of the Hartree-Fock approximation. However, the value of $\Delta \rho$ for every approximate form shows a certain value that is not always negligible. Given the fact that the total energy is reproduced with enough accuracy, the electron density and the total energy cannot be simultaneously reproduced by the present scheme including approximate forms (i)-(vi). This seems to indicate a sort of the limitations.

In order to evaluate the abilities and limitations of the present scheme in more details, we calculate the virial ratio, the kinetic energy, and the potential energy that is defined as the sum of the external potential and the electron-electron interaction energies. The ratio of the kinetic energy (or potential energy) to the corresponding result of the CI method is shown in Table II. The kinetic energy of the CI method is estimated under the assumption that the virial ratio is equal to -2.0. It is found from Table II that every case exhibits the same tendency, that is, underestimation of the kinetic (potential) energy by about $4.4 \%(2.2 \%)$, and overestimation of the virial ratio by about $2.3 \%$. These errors indicate the limitations of the present scheme including approximate forms (i)(vi).

The approximate forms (i)-(vi) are developed by using two restrictive conditions. Of course, the exact kinetic energy functional exists in the set of functionals that satisfy these two restrictive conditions. In Sec. IV, approximate forms are limited to the form of Eq. (31) with $f\left(\mathbf{r}, \mathbf{r}^{\prime}\right)$ that is given as a solution of Eq. (33). Since Eq. (33) is a sufficient condition for satisfying the scaling property, six approximate forms [(i)-(vi)] belong to a subset of the functionals that satisfy two restrictive conditions (see, Fig. 2). Therefore, the abovementioned common errors would primarily be caused from the limitations on the functionals that belong to such a subset. We first have to develop and use the functionals belonging to the set A instead of functionals of the set B (Fig. 2).

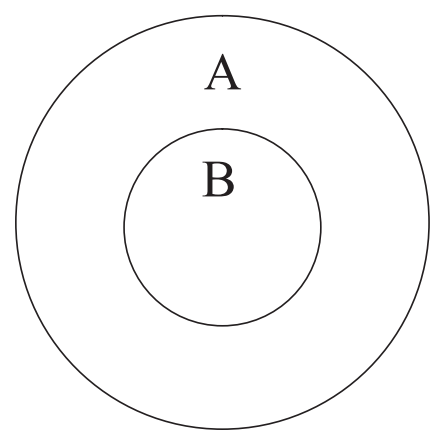

FIG. 2. The sets of approximate functionals of the kinetic energy are illustrated. The set A consists of all of the approximate kinetic energy functionals which satisfy two restrictive conditions given by Eqs. (26) and (30). The set B is the subset of A, which consists of the approximate kinetic energy functionals we have developed under the condition of Eq. (33).

The other possible cause of the common errors would be associated with the present search region of the PDs. For details on this issue, we shall comment in the following section.

\section{Features of the present scheme}

In order to clarify the position of the present scheme in the field of the PD functional theory, we shall mention the features of the present scheme.

(1) The search region of the PDs is definitely extended beyond that of the effective initial theory. ${ }^{20,22,23}$ Therefore, there is no question that the resultant PD can reproduce the correlation effects more than that of the effective initial theory. Of course, it is difficult to clarify to what extent the PDs of the present scheme, which have the below-mentioned feature (feature 3), cover the full search region of the $N$-representable PD's but it is of great worth to check how the best PD of the present scheme is close to the exact one from the viewpoint of the progress of the computational PD functional theory.

(2) The present scheme utilizes several types of approximate functionals of the kinetic energy which have been developed with the aid of two restrictive conditions derived in this work. As already mentioned in Sec. IV, these approximate functionals belong to the subset B (Fig. 2). If all of the functionals of the set A were to be attempted, we can necessarily obtain the best PD within the present search region of the PDs. Namely, the best PD of the extended search region of the present scheme can be obtained only by attempting all functionals of the set A. Therefore, we cannot judge whether the search region of the PDs of the present scheme may be smaller than the set of the $N$-representable PDs unless the above-mentioned best PD is obtained. For this reason, what is to be done primarily is to promote further development of the approximate form of the kinetic energy functional.

(3) There is a possibility that the correlation effects are effectively included into the resultant PD by using only singly-excited SDs. This is different from the case of the CI method of the wave function theory, where Brillouin's theorem holds and doubly-excited SDs are indispensable to de- 


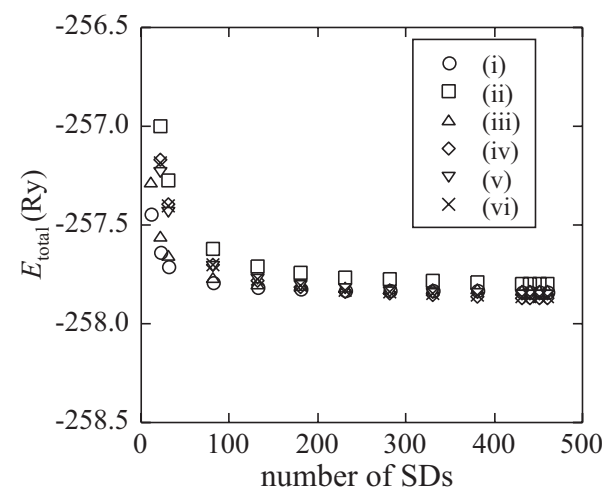

FIG. 3. Dependence of the total energy $\left(E_{\text {total }}\right)$ on the number of the singly-excited SDs.

scribe the correlation effects. ${ }^{66}$ This difference comes from the following two facts. One is that the set of SDs are constructed from the single-particle orbitals that are calculated by the effective initial scheme. ${ }^{22,23}$ The other is that we necessarily utilize the approximate functional $T\left[\gamma^{(2)}\right]$ in calculating the kinetic energy contribution to matrix elements. Due to these facts, it would be expected that the PDs which are constructed by using singly-excited SDs may effectively reproduce the correlation effects.

Taking these features into consideration, the future works will have to be done in the following order:

(1) First, we will reconsider the approximate forms by using directly Eq. (30) or (32) instead of by using Eq. (33). We should normally develop the approximate forms on the basis of Eq. (30) or (32) with a minimum limitation so as to attempt a lot of approximate functionals belonging to the set A.

(2) Second, we could have a way to derive the other restrictive conditions on the kinetic energy functional. Using them, different types of approximate functionals from the present ones [(i)-(vi)] may be obtained. These different functionals might yield the PD that is more close to the best PD than that of the present scheme. Of course, any restrictive condition necessarily leads to the best PD of the present search region if all of the approximate functionals that satisfy it were to be tested. However, it would be impossible to derive all of the approximate functionals that satisfy some restrictive condition. Therefore, it seems to be significant from the practical viewpoint to find and adopt the restrictive condition that can lead to the best PD more efficiently. In this sense, it would also be a meaningful future work to attempt the existing approximate forms ${ }^{16,30,41,43}$ after checking which restrictive conditions are fulfilled by them.

(3) If it is confirmed as a result of the above approaches 1 and 2 that the search region of the PDs of the present scheme is comparatively small, then the search region of the PDs has to be extended beyond the present one. Figure 3 shows the dependence of the total energy on the number of the singlyexcited SDs. The convergence is obtained by using more than 400 singly-excited SDs. Thus, the search region of the PDs cannot be substantially extended only by increasing the number of singly-excited SDs in Eq. (1). We then need to take doubly- or more excited SDs in constructing the PD.
From the above considerations, the priorities of the approaches 1 and 2 would be higher than that of the approach 3.

\section{CONCLUDING REMARKS}

We have presented a computational PD functional theory, in which PDs are reproduced by means of multiple SDs. This theory has desired features that the $N$-representable and correlated PDs can be obtained beyond the effective initial scheme by utilizing the approximate kinetic energy functionals developed here.

Concerning approximate forms of the kinetic energy functional, we have first derived two kinds of restrictive conditions on them. One is derived by considering the physical meaning of the Lagrange multiplier and the other is derived from the scaling property of the kinetic energy functional. These restrictive conditions are effectively used in developing approximate forms of $T\left[\gamma^{(2)}\right]$. Imposing such two restrictive conditions on approximate forms, we have developed six kinds of approximate forms.

We have also checked the abilities and limitations of the present scheme including such approximate forms by calculating the electronic structure of the neutral neon atom. The total energy is reproduced beyond the effective initial scheme while keeping the electron density within the accuracy better than the Hartree-Fock approximation. This means that correlation effects are reasonably incorporated into the resultant PDs. However, it is also shown that the present scheme exhibits a sort of limitations, to be more precise, it yields errors of the electron density, kinetic energy and potential energy at a little but uniform rate. These limitations suggest not only the necessity for developing more appropriate approximation of $T\left[\gamma^{(2)}\right]$ but also the possibility that the $N$-representable and correlated PD which causes less errors can be obtained if we will do the future works shown in the preceding section.

Thus, the present scheme is positioned above the effective initial scheme $e^{20,22,23}$ and offers a promising direction to the progress of the computational PD functional theory.

\section{ACKNOWLEDGMENTS}

This work was partially supported by Grant-in-Aid for Scientific Research (Grants No. 19540399 and No. 22540390) of the Ministry of Education, Culture, Sports, Science, and Technology, Japan.

\section{APPENDIX: EXPRESSIONS FOR THE MATRIX ELEMENTS $A_{i j}$}

The matrix elements $A_{i j}$ can be further rewritten by using single-particle orbitals that are given by

$$
\phi_{n l m \sigma}^{i}(\mathbf{r} \eta)=\frac{P_{n l}^{i}(r)}{r} Y_{l m}(\hat{r}) \chi_{\sigma}(\eta),
$$

where $\phi_{n l m \sigma}^{i}(\mathbf{r} \eta)$ is the constituent orbital of $\Phi_{i}$, and where $P_{n l}^{i}(r), Y_{l m}(\hat{r})$, and $\chi_{\sigma}(\eta)$ stand for a radial wave function multiplied by $r$, spherical harmonics, and spin function, 
respectively. By substituting Eq. (A1) into Eq. (49), the electron-electron interactions of Eq. (49), which have three cases, are respectively, rewritten by

$$
\begin{aligned}
& e^{2} \iint \frac{\gamma_{\mathrm{SSD}, i}^{(2)}\left(\mathbf{r r}^{\prime} ; \mathbf{r r}^{\prime}\right)}{\left|\mathbf{r}-\mathbf{r}^{\prime}\right|} d^{3} r d^{3} r^{\prime}=\sum_{L} \sum_{\alpha} \sum_{\beta} \frac{2 \pi e^{2}}{2 L+1}(-1)^{m_{\alpha}+m_{\beta}}\left\{I_{i i}^{1}(L, \alpha, \alpha, \beta, \beta)-\delta_{\sigma_{\alpha}, \sigma_{\beta}} I_{i i}^{1}(L, \alpha, \beta, \beta, \alpha)\right\} \quad(i=j), \\
& e^{2} \iint \frac{\Gamma_{i_{1} j_{1}}^{i j}\left(\mathbf{r r}^{\prime}\right)}{\left|\mathbf{r}-\mathbf{r}^{\prime}\right|} d^{3} r d^{3} r^{\prime}=\sum_{L} \sum_{\beta}^{N-1} \frac{4 \pi e^{2}}{2 L+1}(-1)^{\bar{n}_{i_{1}}+\bar{n}_{j_{1}}+m_{i_{1}}+m_{\beta}}\left\{\delta_{\sigma_{i_{1}}, \sigma_{j_{1}}} I_{i j}^{1}\left(L, i_{1}, j_{1}, \beta, \beta\right)-\delta_{\sigma_{i_{1}}, \sigma_{\beta}} \delta_{\sigma_{\beta}, \sigma_{j_{1}}} I_{i j}^{1}\left(L, i_{1}, \beta, \beta, j_{1}\right)\right. \\
& \left.-\delta_{\sigma_{\beta}, \sigma_{j_{1}}} \delta_{\sigma_{i_{1}}, \sigma_{\beta}} I_{i j}^{1}\left(L, \beta, j_{1}, i_{1}, \beta\right)+\delta_{\sigma_{i_{1}}, \sigma_{j_{1}}} I_{i j}^{1}\left(L, \beta, \beta, i_{1}, j_{1}\right)\right\} \quad\left(i \neq{ }^{1} j\right),
\end{aligned}
$$

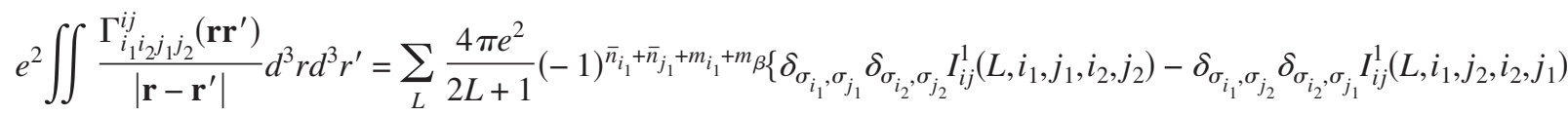

$$
\begin{aligned}
& \left.-\delta_{\sigma_{i_{2}}, \sigma_{j_{1}}} \delta_{\sigma_{i_{1}}, \sigma_{j_{2}}} I_{i j}^{1}\left(L, i_{2}, j_{1}, i_{1}, j_{2}\right)+\delta_{\sigma_{i_{2}}, \sigma_{j_{2}}} \delta_{\sigma_{i_{1}}, \sigma_{j_{1}}} I_{i j}^{1}\left(L, i_{2}, j_{2}, i_{1}, j_{1}\right)\right\} \quad\left(i \neq \neq^{2} j\right)
\end{aligned}
$$

with

$$
\begin{aligned}
I_{i j}^{1}(L, p, q, r, s)= & \delta_{m_{q}-m_{p}, m_{r}-m_{s}}(-1)^{m_{p}-m_{q}} \frac{(2 L+1) \sqrt{\left(2 l_{p}+1\right)\left(2 l_{q}+1\right)\left(2 l_{r}+1\right)\left(2 l_{s}+1\right)}}{4 \pi}\left(\begin{array}{ccc}
l_{p} & l_{q} & L \\
0 & 0 & 0
\end{array}\right)\left(\begin{array}{ccc}
l_{r} & l_{s} & L \\
0 & 0 & 0
\end{array}\right) \\
& \times\left(\begin{array}{ccc}
l_{p} & l_{q} & L \\
-m_{p} & m_{q} & m_{p}-m_{q}
\end{array}\right)\left(\begin{array}{ccc}
l_{r} & l_{s} & L \\
-m_{r} & m_{s} & m_{r}-m_{s}
\end{array}\right) \int_{0}^{\infty} \int_{0}^{\infty} \frac{r_{<}^{L}}{r_{>}^{L+1}} P_{n_{p} l_{p}}^{i}(r)^{*} P_{n_{q} l_{q}}^{j}(r) P_{n_{r} l_{r}}^{i}\left(r^{\prime}\right)^{*} P_{n_{s} l_{s}}^{j}\left(r^{\prime}\right) d r d r^{\prime},
\end{aligned}
$$

where $\left(\begin{array}{lll}a & b & c \\ d & e & f\end{array}\right)$ denotes the Wigner $3 j$ symbol. ${ }^{67}$ Similarly, the external potential energies of Eq. (49), which also have three cases, are respectively, rewritten by

$$
\begin{aligned}
& \iint \frac{v_{e x t}(\mathbf{r})+v_{e x t}\left(\mathbf{r}^{\prime}\right)}{N-1} \gamma_{\mathrm{SSD}, i}^{(2)}\left(\mathbf{r r}^{\prime} ; \mathbf{r r}^{\prime}\right) d^{3} r d^{3} r^{\prime}=\int_{0}^{\infty} v_{e x t}(r) \sum_{\alpha}\left|P_{n_{\alpha^{l} \alpha}}^{i}(r)\right|^{2} d r \quad(i=j),
\end{aligned}
$$

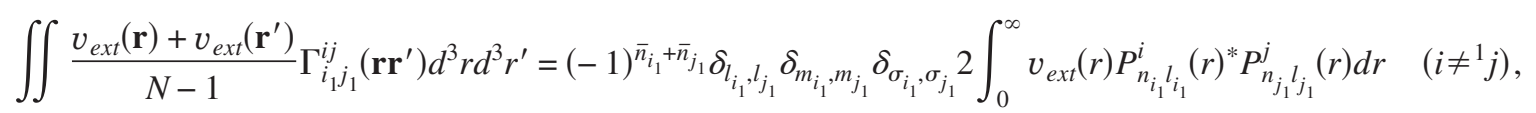

$$
\begin{aligned}
& \iint \frac{v_{e x t}(\mathbf{r})+v_{e x t}\left(\mathbf{r}^{\prime}\right)}{N-1} \Gamma_{i_{1} i_{2} j_{1} j_{2}}^{i j}\left(\mathbf{r r}^{\prime}\right) d^{3} r d^{3} r^{\prime}=0 \quad\left(i \neq \neq^{2} j\right) .
\end{aligned}
$$

The kinetic energy terms of Eq. (49) are varied depending on which functions are chosen as $f_{\mu}\left(\mathbf{r}, \mathbf{r}^{\prime}\right)$ and $f_{\nu}\left(\mathbf{r}, \mathbf{r}^{\prime}\right)$. In what follows, we shall illustrate by an example the kinetic energy terms of the approximate form given by Eq. (45). Their matrix elements for three cases are respectively given by

$$
\begin{aligned}
& \iint\left\{\frac{K}{\left|\mathbf{r}-\mathbf{r}^{\prime}\right|^{2}}+\frac{K^{\prime}}{\sqrt{r r^{\prime}}}\left(\frac{1}{r}+\frac{1}{r^{\prime}}\right)\right\} \gamma_{\mathrm{SSD}, i}^{(2)}\left(\mathbf{r r}^{\prime} ; \mathbf{r r}^{\prime}\right) d^{3} r d^{3} r^{\prime}=\sum_{L} \sum_{L^{\prime}} \sum_{\alpha} \sum_{\beta} \frac{8 \pi^{2} K(-1)^{m_{\alpha}+m_{\beta}}}{(2 L+1)\left(2 L^{\prime}+1\right)}\left\{I_{i i}^{2}\left(L, L^{\prime}, \alpha, \alpha, \beta, \beta\right)\right. \\
& \left.-\delta_{\sigma_{\alpha}, \sigma_{\beta}} I_{i i}^{2}\left(L, L^{\prime}, \alpha, \beta, \beta, \alpha\right)\right\}+K^{\prime} \sum_{\alpha} \sum_{\beta}\left\{I_{i i}^{3}(\alpha, \alpha, \beta, \beta)\right. \\
& \left.-I_{i i}^{3}(\alpha, \beta, \beta, \alpha)\right\} \quad(i=j), \\
& \iint\left\{\frac{K}{\left|\mathbf{r}-\mathbf{r}^{\prime}\right|^{2}}+\frac{K^{\prime}}{\sqrt{r r^{\prime}}}\left(\frac{1}{r}+\frac{1}{r^{\prime}}\right)\right\} \Gamma_{i_{1} j_{1}}^{i j}\left(\mathbf{r r}^{\prime}\right) d^{3} r d^{3} r^{\prime}=\sum_{L} \sum_{L^{\prime}} \sum_{\beta}^{N-1} \frac{16 \pi^{2} K(-1)^{\bar{n}_{1}+\bar{n}_{j_{1}}+m_{i_{1}}+m_{\beta}}}{(2 L+1)\left(2 L^{\prime}+1\right)}\left\{\delta_{\sigma_{i_{1}}, \sigma_{j_{1}}} I_{i j}^{2}\left(L, L^{\prime}, i_{1}, j_{1}, \beta, \beta\right)\right. \\
& -\delta_{\sigma_{i_{1}}, \sigma_{\beta}} \delta_{\sigma_{\beta}, \sigma_{j_{1}}} I_{i j}^{2}\left(L, L^{\prime}, i_{1}, \beta, \beta, j_{1}\right)-\delta_{\sigma_{\beta}, \sigma_{j_{1}}} \delta_{\sigma_{i_{1}}, \sigma_{\beta}} I_{i j}^{2}\left(L, L^{\prime}, \beta, j_{1}, i_{1}, \beta\right) \\
& \left.+\delta_{\sigma_{i_{1}}, \sigma_{j_{1}}} I_{i j}^{2}\left(L, L^{\prime}, \beta, \beta, i_{1}, j_{1}\right)\right\}+K^{\prime} \sum_{\beta}^{N-1}(-1)^{\bar{n}_{i_{1}}+\bar{n}_{j}}\left\{I_{i j}^{3}\left(i_{1}, j_{1}, \beta, \beta\right)\right. \\
& \left.-I_{i j}^{3}\left(i_{1}, \beta, \beta, j_{1}\right)-I_{i j}^{3}\left(\beta, j_{1}, i_{1}, \beta\right)+I_{i j}^{3}\left(\beta, \beta, i_{1}, j_{1}\right)\right\} \quad\left(i \neq{ }^{1} j\right),
\end{aligned}
$$




$$
\begin{aligned}
& \iint\left\{\frac{K}{\left|\mathbf{r}-\mathbf{r}^{\prime}\right|^{2}}+\frac{K^{\prime}}{\sqrt{r r^{\prime}}}\left(\frac{1}{r}+\frac{1}{r^{\prime}}\right)\right\} \Gamma_{i_{1} i_{2} j_{1} j_{2}}^{i j}\left(\mathbf{r r}^{\prime}\right) d^{3} r d^{3} r^{\prime}=\sum_{L} \sum_{L^{\prime}} \frac{16 \pi^{2} K(-1)^{\bar{n}_{i_{1}}+\bar{n}_{i_{2}}\left(i_{1}\right)+\bar{n}_{j_{1}}+\bar{n}_{j_{2}}\left(j_{1}\right)+m_{i_{1}}+m_{i_{2}}}}{(2 L+1)\left(2 L^{\prime}+1\right)} \\
& \times\left\{\delta_{\sigma_{i_{1}}, \sigma_{j_{1}}} \delta_{\sigma_{i_{2}}, \sigma_{j_{2}}} I_{i j}^{2}\left(L, L^{\prime}, i_{1}, j_{1}, i_{2}, j_{2}\right)-\delta_{\sigma_{i_{1}}, \sigma_{j_{2}}} \delta_{\sigma_{i_{2}}, \sigma_{j_{1}}} I_{i j}^{2}\left(L, L^{\prime}, i_{1}, j_{2}, i_{2}, j_{1}\right)\right. \\
& \left.-\delta_{\sigma_{i_{2}}, \sigma_{j_{1}}} \delta_{\sigma_{i_{1}}, \sigma_{j_{2}}} I_{i j}^{2}\left(L, L^{\prime}, i_{2}, j_{1}, i_{1}, j_{2}\right)+\delta_{\sigma_{i_{2}}, \sigma_{j_{2}}} \delta_{\sigma_{i_{1}}, \sigma_{j_{1}}} I_{i j}^{2}\left(L, L^{\prime}, i_{2}, j_{2}, i_{1}, j_{1}\right)\right\} \\
& +K^{\prime}(-1)^{\bar{n}_{i_{1}}+\bar{n}_{i_{2}}\left(i_{1}\right)+\bar{n}_{j_{1}}+\bar{n}_{j_{2}}\left(j_{1}\right)}\left\{I_{i j}^{3}\left(i_{1}, j_{1}, i_{2}, j_{2}\right)-I_{i j}^{3}\left(i_{1}, j_{2}, i_{2}, j_{1}\right)-I_{i j}^{3}\left(i_{2}, j_{1}, i_{1}, j_{2}\right)\right. \\
& \left.+I_{i j}^{3}\left(i_{2}, j_{2}, i_{1}, j_{1}\right)\right\} \quad\left(i \neq \neq^{2} j\right)
\end{aligned}
$$

with

$$
\begin{aligned}
& I_{i j}^{2}\left(L, L^{\prime}, p, q, r, s\right)=\delta_{m_{q}-m_{p}, m_{r}-m_{s}}(-1)^{L+L^{\prime}+L_{a}+m_{p}-m_{q}} \sum_{L_{a}=\left|L-L^{\prime}\right|}^{L+L^{\prime}} \sum_{L_{b}=\left|l_{p}-l_{q}\right|}^{l_{p}+l_{q}} \sum_{L_{c}=\left|l_{r}-l_{s}\right|}^{l_{r}+l_{s}} \delta_{L_{a}, L_{b}} \delta_{L_{a}, L_{c}} \\
& \times \frac{(2 L+1)\left(2 L^{\prime}+1\right) \sqrt{\left(2 l_{p}+1\right)\left(2 l_{q}+1\right)\left(2 l_{r}+1\right)\left(2 l_{s}+1\right)\left(2 L_{b}+1\right)\left(2 L_{c}+1\right)}}{16 \pi^{2}}\left(\begin{array}{lll}
L & L^{\prime} & L_{a} \\
0 & 0 & 0
\end{array}\right)^{2}\left(\begin{array}{lll}
l_{p} & l_{q} & L_{b} \\
0 & 0 & 0
\end{array}\right) \\
& \times\left(\begin{array}{ccc}
l_{r} & l_{s} & L_{c} \\
0 & 0 & 0
\end{array}\right)\left(\begin{array}{ccc}
l_{p} & l_{q} & L_{b} \\
-m_{p} & m_{q} & m_{p}-m_{q}
\end{array}\right)\left(\begin{array}{ccc}
l_{r} & l_{s} & L_{c} \\
-m_{r} & m_{s} & m_{r}-m_{s}
\end{array}\right) \\
& \times \int_{0}^{\infty} \int_{0}^{\infty} \frac{r_{<}^{L+L^{\prime}}}{r_{>}^{L+L^{\prime}+2}} P_{n_{p} l_{p}}^{i}(r)^{*} P_{n_{q} l_{q}}^{j}(r) P_{n_{r} l_{r}}^{i}\left(r^{\prime}\right)^{*} P_{n_{s} l_{s}}^{j}\left(r^{\prime}\right) d r d r^{\prime}
\end{aligned}
$$

and

$$
I_{i j}^{3}(p, q, r, s)=\delta_{l_{p}, l_{q}} \delta_{m_{p}, m_{q}} \delta_{\sigma_{p}, \sigma_{q}} \delta_{l_{r}, l_{s}} \delta_{m_{r}, m_{s}} \delta_{\sigma_{r}, \sigma_{s}} \int_{0}^{\infty} \int_{0}^{\infty} \frac{1}{r \sqrt{r r^{\prime}}} P_{n_{p} l_{p}}^{i}(r)^{*} P_{n_{q} l_{q}}^{j}(r) P_{n_{r} l_{r}}^{i}\left(r^{\prime}\right)^{*} P_{n_{s} l_{s}}^{j}\left(r^{\prime}\right) d r d r^{\prime}
$$

${ }^{1}$ R. G. Parr and W. Yang, Density-Functional Theory of Atoms and Molecules (Oxford University Press, New York, 1989), Chap. 2.

${ }^{2}$ A. J. Coleman, Rev. Mod. Phys. 35, 668 (1963).

${ }^{3}$ A. J. Coleman, in The Force Concept in Chemistry, edited by B. M. Deb (Van Nostrand Reinhold, New York, 1981), p. 418.

${ }^{4}$ P. Hohenberg and W. Kohn, Phys. Rev. 136, B864 (1964).

${ }^{5}$ W. Kohn and L. J. Sham, Phys. Rev. 140, A1133 (1965).

${ }^{6}$ P. Ziesche, Phys. Lett. A 195, 213 (1994).

${ }^{7}$ P. Ziesche, Int. J. Quantum Chem. 60, 1361 (1996).

${ }^{8}$ A. Gonis, T. C. Schulthess, J. van Ek, and P. E. A. Turchi, Phys. Rev. Lett. 77, 2981 (1996).

${ }^{9}$ A. Gonis, T. C. Schulthess, P. E. A. Turchi, and J. van Ek, Phys. Rev. B 56, 9335 (1997).

${ }^{10}$ M. Levy and P. Ziesche, J. Chem. Phys. 115, 9110 (2001).

${ }^{11}$ F. Furche, Phys. Rev. A 70, 022514 (2004).

${ }^{12}$ Á. Nagy, Phys. Rev. A 66, 022505 (2002).

${ }^{13}$ Á. Nagy and C. Amovilli, J. Chem. Phys. 121, 6640 (2004).

${ }^{14}$ B. Hetényi, L. Brualla, and S. Fantoni, Phys. Rev. Lett. 93, 170202 (2004).

${ }^{15}$ J. K. Percus, J. Chem. Phys. 122, 234103 (2005).

${ }^{16}$ P. W. Ayers, J. Math. Phys. 46, 062107 (2005).

${ }^{17}$ P. W. Ayers and M. Levy, J. Chem. Sci. 117, 507 (2005).

${ }^{18}$ Á. Nagy, Int. J. Quantum Chem. 106, 1043 (2006).

${ }^{19}$ P. W. Ayers, S. Golden, and M. Levy, J. Chem. Phys. 124,
054101 (2006).

${ }^{20}$ M. Higuchi and K. Higuchi, Physica B 387, 117 (2007).

${ }^{21}$ M. Higuchi and K. Higuchi, Phys. Rev. A 75, 042510 (2007).

${ }^{22}$ M. Higuchi and K. Higuchi, Phys. Rev. B 78, 125101 (2008).

${ }^{23}$ K. Higuchi and M. Higuchi, J. Phys.: Condens. Matter 21, 064206 (2009).

${ }^{24}$ Many-Electron Densities and Reduced Density Matrices, edited by J. Closlowski (Kluwer, Dordrecht/Plenum, New York, 2000).

${ }^{25}$ A. J. Coleman and V. I. Yukalov, Reduced Density Matrices: Coulson's Challenge (Springer-Verlag, Berlin, 2000).

${ }^{26}$ The Fundamentals of Electron Density in Density Matrix and Density Functional Theory in Atoms, Molecules and the Solid States, edited by N. I. Gidopoulos and S. Wilson (Kluwer, New York, 2003).

${ }^{27}$ E. R. Davidson, Chem. Phys. Lett. 246, 209 (1995).

${ }^{28}$ S. Kh. Samvelyan, Int. J. Quantum Chem. 65, 127 (1997).

${ }^{29}$ M.-E. Pistol, Chem. Phys. Lett. 400, 548 (2004).

${ }^{30}$ P. W. Ayers and M. Levy, Chem. Phys. Lett. 415, 211 (2005).

${ }^{31}$ P. W. Ayers and E. R. Davidson, Int. J. Quantum Chem. 106, 1487 (2006).

${ }^{32}$ P. Gori-Giorgi and A. Savin, Philos. Mag. 86, 2643 (2006).

${ }^{33}$ M.-E. Pistol, Chem. Phys. Lett. 417, 521 (2006).

${ }^{34}$ M.-E. Pistol, Chem. Phys. Lett. 422, 363 (2006).

${ }^{35}$ M.-E. Pistol, Chem. Phys. Lett. 431, 216 (2006).

${ }^{36}$ P. W. Ayers, Phys. Rev. A 74, 042502 (2006). 
${ }^{37}$ P. W. Ayers and S. Liu, Phys. Rev. A 75, 022514 (2007).

${ }^{38}$ P. W. Ayers and E. R. Davidson, Adv. Chem. Phys. 134, 443 (2007).

${ }^{39}$ M.-E. Pistol, Chem. Phys. Lett. 449, 208 (2007).

${ }^{40}$ B. Hetényi and A. W. Hauser, Phys. Rev. B 77, 155110 (2008).

${ }^{41}$ R. Cuevas-Saavedra and P. W. Ayers, Int. J. Quantum Chem. 109, 1699 (2009).

${ }^{42}$ As an approach beyond the effective initial scheme, which is different from the scheme mentioned in this paper, we have recently proposed the PD functional theory utilizing the Jastrow wave function (Ref. 21). However, this theory is not free from the $N$-representability problem of the PD.

${ }^{43}$ N. H. March and R. Santamaria, Int. J. Quantum Chem. 39, 585 (1991).

${ }^{44}$ J. P. Perdew and Y. Wang, Phys. Rev. B 33, 8800 (1986).

${ }^{45}$ J. P. Perdew, in Electronic Structure of Solids '91, edited by P. Ziesche and H. Eschrig (Akademie Verlag, Berlin, 1991), p. 11.

${ }^{46}$ J. P. Perdew, K. Burke, and M. Ernzerhof, Phys. Rev. Lett. 77, 3865 (1996).

${ }^{47}$ J. P. Perdew, A. Ruzsinszky, G. I. Csonka, O. A. Vydrov, G. E. Scuseria, L. A. Constantin, X. Zhou, and K. Burke, Phys. Rev. Lett. 100, 136406 (2008); 102, 039902(E) (2009).

${ }^{48}$ K. Higuchi and M. Higuchi, Phys. Rev. B 74, 195122 (2006); 75, 159902(E) (2007).

${ }^{49}$ M. Higuchi and K. Higuchi, Phys. Rev. B 75, 195114 (2007).

${ }^{50} \mathrm{~K}$. Higuchi and M. Higuchi, J. Phys.: Condens. Matter 19, 365216 (2007).
${ }^{51}$ M. Higuchi and K. Higuchi, Phys. Rev. A 81, 042505 (2010).

${ }^{52}$ M. Levy, Proc. Natl. Acad. Sci. U.S.A. 76, 6062 (1979).

${ }^{53}$ E. H. Lieb, Int. J. Quantum Chem. 24, 243 (1983); Density Functional Methods in Physics, NATO Advanced Study Institute, Series B: Physics Vol. 123, edited by R. M. Dreizler and J. da Providencia (Plenum, New York, 1985), p. 31; also see Physics as Natural Philosophy, edited by A. Shimony and H. Freshbach (MIT Press, Cambridge, MB, 1982), p. 111.

${ }^{54}$ M. Levy, Phys. Rev. A 26, 1200 (1982).

${ }^{55}$ M. Higuchi and K. Higuchi, Phys. Rev. B 69, 035113 (2004).

${ }^{56}$ K. Higuchi and M. Higuchi, Phys. Rev. B 69, 165118 (2004).

${ }^{57}$ K. Higuchi and M. Higuchi, Phys. Rev. B 71, 035116 (2005).

${ }^{58}$ Á. Nagy, S. Liu, and L. Bartolloti, J. Chem. Phys. 122, 134107 (2005).

${ }^{59}$ P. W. Ayers and P. Fuentealba, Phys. Rev. A 80, 032510 (2009).

${ }^{60}$ K. Higuchi and M. Higuchi, Phys. Rev. A 79, 022113 (2009).

${ }^{61}$ A. V. Bunge and R. O. Esquivel, Phys. Rev. A 34, 853 (1986).

${ }^{62}$ A. Veillard and E. Clementi, J. Chem. Phys. 49, 2415 (1968).

${ }^{63}$ S. E. Koonin, Computational Physics (Addison-Wesley, Reading, MA, 1986).

${ }^{64}$ E. Engel, H. Jiang, and A. F. Bonetti, Phys. Rev. A 72, 052503 (2005).

${ }^{65}$ H. Jiang and E. Engel, J. Chem. Phys. 123, 224102 (2005).

${ }^{66}$ A. Szabo and N. Ostlund, Modern Quantum Chemistry, 1st ed. (Dover, New York, 1996).

${ }^{67}$ A. Messiah, Quantum Mechanics (Dover, New York, 1999). 